\title{
La comunicación estratégica como articulador de los intangibles organizacionales
}

\section{Strategic Communication as an Articulator of Organizational Intangibles}

\section{A comunicação estratégica como articuladora dos intangíveis organizacionais}

\section{Patricia Durán Bravo} Benemérita Universidad Autónoma de Puebla, Facultad de Ciencias de la Comunicación, México

patricia.duran@correo.buap.mx https://orcid.org/0000-0003-2388-2432

Víctor Meléndez Rodríguez

Benemérita Universidad Autónoma de Puebla, Facultad de Ciencias de la Comunicación, México victor.melendez@correo.buap.mx https://orcid.org/0000-0002-9518-3019

Rafael Cid Quiroz Benemérita Universidad Autónoma de Puebla, Facultad de Ciencias de la Comunicación, México rafael.cidquiroz@correo.buap.mx https://orcid.org/0000-0001-6253-1526 


\section{Revista Iberoamericana \\ de las Ciencias Sociales y \\ Humanísticas}

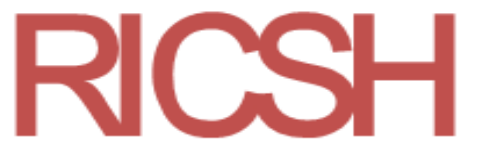

ISSN: 2395 - 7972

\section{Resumen}

La economía del conocimiento ha orillado a las corporaciones a cambiar la manera de organizarse para propiciar mayor rentabilidad, efectividad y diferenciación. Un aspecto fundamental de esta transición es replantear el cálculo del patrimonio organizacional considerando los activos intangibles. La comunicación estratégica es un proceso generador de energía simbólica, movimiento relacional y acción social, por lo que su función es precisamente la generación de activos intangibles dentro del espectro organizacional. El objetivo de este análisis es hacer una revisión documental para identificar los consensos teóricos del concepto activos intangibles y así dar con la utilidad de estos y la forma en que se generan. Los resultados ayudarán a identificar el papel que juegan los procesos comunicacionales en la producción de este tipo de activos y, finalmente, permitirán orientar las estrategias comunicativas en las organizaciones que favorezcan su producción para alcanzar la generación de valor comunicacional y la competitividad organizacional.

Palabras clave: activos intangibles, comunicación estratégica, gestión, patrimonio organizacional.

\section{Abstract}

The knowledge economy has led corporations to change the way they organize themselves to promote greater profitability, effectiveness and differentiation. A fundamental aspect of this transition is to rethink the calculation of organizational assets considering intangible assets. Strategic communication is a process that generates symbolic energy, relational movement and social action, so its function is precisely the generation of intangible assets within the organizational spectrum. The objective of this analysis is to carry out a documentary review to identify the theoretical consensuses of the intangible assets concept and thus find the usefulness of these and the way in which they are generated. The results will help to identify the role that communicational processes play in the production of this type of assets and, finally, will allow to orient communication strategies in organizations that favor their production to achieve the generation of communicational value and organizational competitiveness.

Keywords: intangible assets, strategic communication, management, organizational heritage. 


\section{Revista Iberoamericana \\ de las Ciencias Sociales y \\ Humanísticas}

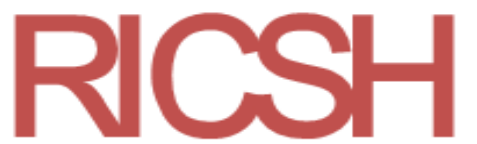

ISSN: 2395 - 7972

\section{Resumo}

A economia do conhecimento tem levado as empresas a mudar a forma como se organizam para promover maior lucratividade, eficácia e diferenciação. Um aspecto fundamental dessa transição é repensar o cálculo dos ativos organizacionais considerando os ativos intangíveis. A comunicação estratégica é um processo que gera energia simbólica, movimento relacional e ação social, portanto sua função é justamente a geração de ativos intangíveis dentro do espectro organizacional. O objetivo desta análise é realizar uma revisão documental para identificar os consensos teóricos do conceito de ativos intangíveis e, assim, verificar a utilidade destes e a forma como são gerados. Os resultados ajudarão a identificar o papel que os processos comunicacionais desempenham na produção deste tipo de ativos e, por fim, permitirão orientar estratégias de comunicação nas organizações que favoreçam sua produção para a geração de valor comunicacional e competitividade organizacional.

Palavras-chave: ativos intangíveis, comunicação estratégica, gestão, patrimônio organizacional.

Fecha Recepción: Octubre 2019

Fecha Aceptación: Junio 2021

\section{Introducción}

La economía del conocimiento ha provocado un cambio sustancial en la concepción de las organizaciones, que han transitado de sistemas de producción a sistemas de interacción. El mapa relacional en el que se desenvuelven estas interacciones organizacionales se conforma de múltiples y diversos sistemas. Se trata de una diversidad que a su vez forma un macrosistema en el que se articulan dinámicas convergentes y divergentes que se influyen de manera continua, no solo a nivel funcional, sino también a nivel simbólico. Estos escenarios someten a las organizaciones a una permanente tensión rodeada de una gran complejidad. En efecto, las organizaciones se enfrentan, por una parte, a la competitividad técnica, y por otra, a la dinámica social.

En los últimos 50 años, las investigaciones sobre la competitividad de las organizaciones han oscilado entre la importancia de los factores externos, a través de la teoría estructural, y la de los factores internos, con la teoría de recursos y capacidades. La tendencia actual plantea un equilibrio entre ambos paradigmas. Por una parte, la potencialización de los factores internos organizacionales, que se manifiestan en las 


\section{Revista Iberoamericana \\ de las Ciencias Sociales y \\ Humanísticas}

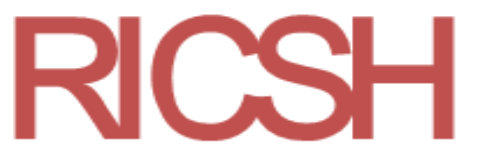

ISSN: $2395-7972$

habilidades del personal, la flexibilidad comercial y las destrezas organizativas, activos intangibles que se generan a partir de las interacciones internas y que resultan fundamentales para dar respuesta a las necesidades del entorno. Por otra parte, las demandas del entorno que afectan las respuestas organizacionales, ya que, en la medida en que se satisfacen dichas demandas, se construye la calidad, la innovación y la sustentabilidad de la organización, otro tipo de activos intangibles que, contrariamente, surgen de la interacción externa y que son igualmente importantes para el desarrollo organizacional.

En este sentido, los paradigmas tradicionales de gestión organizacional han fracasado debido a su enfoque reduccionista. Para lograr la efectividad, es necesario recordar que los sistemas organizacionales son entidades complejas que requieren gestionar sus recursos materiales, financieros y humanos. Para lograr la generación de valor estratégico sustentable, es indispensable integrar los activos intangibles para capitalizar los resultados corporativos. Y para todo lo anterior, se requieren de sistemas de comunicación organizacional que logren una articulación satisfactoria entre los procesos relacionales, culturales y simbólicos.

La competitividad organizacional requiere dejar de lado las tradicionales cadenas de producción, construidas bajo estándares lineales, materiales, operativos y de corto plazo, para dar lugar a redes de colaboración social en donde se considera la incorporación de activos intangibles que aporten beneficios a la red a nivel individual, corporativo y social. Y es particularmente a través de la comunicación estratégica que es posible desarrollar mejores interacciones y relaciones entre todos los componentes sociales de la organización.

La tendencia en los estudios académicos y empíricos apunta a evidenciar el papel creciente de los activos intangibles en la construcción de ventajas competitivas, la innovación productiva, la diferenciación del producto y el aumento de calidad, entre otros. Sin embargo, resulta importante estudiar las perspectivas conceptuales sobre los activos intangibles no solo para expresar sus características, concretar una definición y explicar su medición; comunicacionalmente, es prioritario describir los componentes que intervienen en su proceso de producción y cómo se articulan para contribuir a la competitividad organizacional.

En este sentido, el objetivo de este análisis es hacer una revisión documental para identificar las convergencias conceptuales de los activos intangibles desde una visión comunicacional. Y posteriormente, identificar el papel que juegan los procesos 


\section{Revista Iberoamericana \\ de las Ciencias Sociales y \\ Humanísticas}

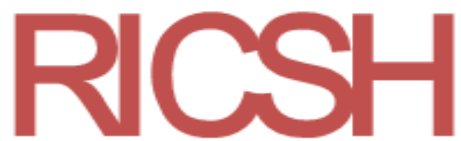

ISSN: $2395-7972$

comunicacionales en la producción de este tipo de activos para orientar las estrategias comunicativas que favorezcan la diferenciación, la generación de valor y la competitividad en las organizaciones.

\section{Metodología}

La presente investigación se desarrolla desde el paradigma cualitativo, pues se centra en aspectos descriptivos. Y recurre a la técnica del análisis de contenido, método que permite identificar las convergencias discursivas en las conceptualizaciones de los activos intangibles de una forma sistemática. En específico, se realizó el análisis de documentos académicos que adoptan la perspectiva de la comunicación estratégica.

El análisis de contenido es una técnica que pretende ser objetiva y sistemática. A través de una serie de instrumentos, permite identificar las unidades y categorías que conforman un discurso; en este caso, se centra en el discurso académico que se construye a partir de las definiciones utilizadas por investigadores que han publicado en revistas académicas en los últimos años.

El objetivo es identificar las redundancias en la conceptualización de los activos intangibles que son preponderantes desde una perspectiva comunicacional. Para ello, se hizo una revisión documental de la producción académica alojada en la base de datos Ebsco-Business Research, en un periodo comprendido entre 2016 al 2021, con el propósito de identificar las tendencias académicas que abordan el tema ya especificado. El resultado de la búsqueda fueron 32 artículos académicos que se presentan en la tabla 1 y 2. 


\section{Revista Iberoamericana \\ de las Ciencias Sociales y \\ Humanísticas}

Tabla 1. Artículos académicos

\begin{tabular}{|c|c|}
\hline Núm. & Lista de resultados (parte 1) \\
\hline 1 & $\begin{array}{l}\text { Loza, I. y Preciado, V. (2020). Contribución de los activos intangibles al valor de } \\
\text { la empresa que cotiza en la Bolsa Mexicana de Valores. Mercados y Negocios, } \\
(42), 75-92 \text {. }\end{array}$ \\
\hline 2 & $\begin{array}{l}\text { Sahut, J. M. et Dang, R. (2020). Introduction au dossier spécial : innovations } \\
\text { digitales et actifs immatériels : quels sont les impacts sur le comportement des } \\
\text { consommateurs et la performance des firmes ? Gestiòn Internacional, 24(5), 100- } \\
105 .\end{array}$ \\
\hline 3 & $\begin{array}{l}\text { Marulanda, N., Rincón, C. y Echeverry, F. (2018). Gestión } \\
\text { de activos intangibles de capital relacional en instituciones de educación superior. } \\
\text { AD-Minister, (33), 85-112. }\end{array}$ \\
\hline 4 & $\begin{array}{l}\text { Rosich, A. (2016). La protección internacional de los activos intangibles. Debates } \\
\text { IESA, 21(2), 10-12. }\end{array}$ \\
\hline 5 & $\begin{array}{l}\text { Gómez, J. A. (2019). El análisis de comparabilidad post-BEPS en precios de } \\
\text { transferencia. una visión sobre las operaciones vinculadas } \\
\text { con activos intangibles. Cizur Menor, España: Aranzadi. }\end{array}$ \\
\hline 6 & $\begin{array}{l}\text { Medeiros, J., Fernandes, H., Ferraz, L. e Louzada, L. (2017). Proposta de } \\
\text { convergência teórica das perspectivas das finanças e da contabilidade na avaliação } \\
\text { de ativos intangíveis. Revista Universo Contábil, 13(4). }\end{array}$ \\
\hline 7 & $\begin{array}{l}\text { Hilahata, I. K., Lien, P., Butarelli, F. P. e Dal Vesco, D. G. (2017). } \\
\text { Desenvolvimento estratégico com base em ativos intangíveis: o caso da empresa } \\
\text { X. Revista Ibero Americana de Estratégia, 16(2), 125-140. }\end{array}$ \\
\hline 8 & $\begin{array}{l}\text { Guimarães, G., Carvalho, L. Maciel, F. e Lima, J. (2020). Governança corporativa } \\
\text { e a intangibilidade: um estudo em empresas brasileiras não financeiras da B3. } \\
\text { Revista Ibero-Americana de Estratégia, 19(4), 58-75 }\end{array}$ \\
\hline 9 & $\begin{array}{l}\text { Orviz, N. y Cuervo, T. (2020). Revisión de la investigación en el ámbito de la } \\
\text { reputación corporativa. } 3 \text { C Empresa, } 9(2) \text {. }\end{array}$ \\
\hline 10 & $\begin{array}{l}\text { Ortiz, N. (2019). Flujo de caja descontado, método financiero para la valuación de } \\
\text { marcas por enfoque de ingresos. Arandu Utic, 6(1), 211-238. }\end{array}$ \\
\hline 11 & $\begin{array}{l}\text { Morales, L., Jacobo, C., Ochoa, S. e Ibarra, L. E. (2019). Capital intelectual y } \\
\text { desempeño organizacional: el caso de las instituciones de educación básica en }\end{array}$ \\
\hline
\end{tabular}


Revista lberoamericana

de las Ciencias Sociales y

Humanísticas

ISSN: 2395 - 7972

\begin{tabular}{|c|l|}
\hline & México. Pensamiento \& Gestión, (47), 180-202. \\
\hline 12 & $\begin{array}{l}\text { Hoag, T. M. e Lemme, C. F. (2018). Indústria de alimentos de origem animal: } \\
\text { riscos e oportunidades para o setor decorrentes das políticas de bem-estar animal. } \\
\text { Revista de Administração de Empresas, 58(3), 244-253. }\end{array}$ \\
\hline 13 & $\begin{array}{l}\text { Contesini, N., Pires, T. y de Paula, C. (2018). Reflexões sobre os aspectos } \\
\text { simbólicos das marcas sob a ótica da teoria institucional. Revista Gestão em } \\
\text { Análise, 7(2), 107. }\end{array}$ \\
\hline 14 & $\begin{array}{l}\text { Palomares, P. y Ripoli, J. (2020). El papel de los incentivos fiscales a la inversión } \\
\text { en I+D+i en España. Boletín Económico de ICE, Información Comercial } \\
\text { Española, (3129), 61-72. }\end{array}$ \\
\hline 15 & $\begin{array}{l}\text { Chang, S. (2019). When to go it alone: Examining post-conversion performance of } \\
\text { international joint ventures. Journal of International Business Studies, 50, 998- } \\
\text { 1020. }\end{array}$ \\
\hline 16 & $\begin{array}{l}\text { Bassi, E., Bittencourt, G., Beuren, I. M., Márcio, L. and Sampaio, G. (2016). } \\
\text { Relationship between organizational slack and innovation in companies of } \\
\text { BM\&FBovespa. Revista de Administração Mackenzie, 17(3). }\end{array}$ \\
\hline
\end{tabular}

Fuente: Elaboración propia

Tabla 2. Artículos académicos 


\section{Revista Iberoamericana \\ de las Ciencias Sociales y \\ Humanísticas}

ISSN: 2395 - 7972

\begin{tabular}{|c|c|}
\hline Núm. & Lista de resultados (parte 2) \\
\hline 17 & $\begin{array}{l}\text { Liu,Y., Jiao, J. and Xia, J. (2019). Subsidiary Networks and Foreign Subsidiary } \\
\text { Performance: A Coopetition Perspective. Management and Organization Review, } \\
\text { 15(1). }\end{array}$ \\
\hline 18 & $\begin{array}{l}\text { João, R., Cipriano, A., Donadio, G. e Silva, F. (2019). Impactos dos investimentos } \\
\text { em inovação e da estrutura de capital no desempenho organizacional: uma análise } \\
\text { sob a perspectiva da equipe de alto escalão. Revista Universo Contábil, 15(2), 59- } \\
77 .\end{array}$ \\
\hline 19 & $\begin{array}{l}\text { Serna, H. y Díaz, A. (2020). Medición del valor del cliente. Desarrollo Gerencial, } \\
\text { 12(1). }\end{array}$ \\
\hline 20 & $\begin{array}{l}\text { Castellani, D. and Lavoratori, K. (2020). The lab and the plant: Offshore R\&D } \\
\text { and co-location with production activities. Journal of International Business } \\
\text { Studies, 51, 121-137. }\end{array}$ \\
\hline 21 & $\begin{array}{l}\text { Navarro, A. (2019). Intangibles de difícil valoración y ajustes retrospectivos en la } \\
\text { normativa española sobre precios de transferencia. Crónica Tributaria, (173), } \\
\text { 159-185. }\end{array}$ \\
\hline 22 & $\begin{array}{l}\text { Cavalcante, J., Silva, T. e Lázaro, J. C. (2016). Analisando as diferenças entre } \\
\text { investimentos em atividades de inovação conforme variáveis estratégicas } \\
\text { contingenciais em empresas de capital aberto no Brasil. Revista Gestão \& } \\
\text { Tecnologia, 16(3). }\end{array}$ \\
\hline 23 & $\begin{array}{l}\text { Henao, M., Rivera, P. and Uribe, B. (2017). Knowledge Management Processes } \\
\text { and Intellectual Property Management Processes: An Integrated Conceptual } \\
\text { Framework. AD-Minister, (31), 137-160. }\end{array}$ \\
\hline 24 & $\begin{array}{l}\text { Beltrán, J. A., López, J. A., Gelvez, C., Quintero, S. y Benítez, V. K. (2019). } \\
\text { Gestión del conocimiento: una estrategia innovadora para el desarrollo de las } \\
\text { universidades. Clío América, 13(26), 362-369. }\end{array}$ \\
\hline 25 & $\begin{array}{l}\text { Belarouci, M., Fonrouge, C. et François, V. (2019). Caractéristiques des jeunes } \\
\text { entreprises innovantes ayant recours au financement participatif : approche } \\
\text { comparative selon les modes de levées de fonds. Revue Internationale P.M.E., } \\
\text { 32(3-4), 63-84. }\end{array}$ \\
\hline 26 & $\begin{array}{l}\text { Marques, R., Gonçalves, V. A. e Camargo, M. E. (2016). O que (ainda) podemos } \\
\text { aprender sobre capacidades dinâmicas. Revista Ibero Americana de Estratégia, }\end{array}$ \\
\hline
\end{tabular}




\section{Revista Iberoamericana \\ de las Ciencias Sociales y \\ Humanísticas}

ISSN: $2395-7972$

\begin{tabular}{|c|c|}
\hline & $15(1), 44-64$ \\
\hline 27 & $\begin{array}{l}\text { Carrillo, M. V. (2016). Importancia del profesional de la información en la } \\
\text { dirección de comunicación de las organizaciones. Profesional de la Información, } \\
25(2), 272-278 .\end{array}$ \\
\hline 28 & $\begin{array}{l}\text { Bernardes, K., Silvestre, A., Brunozi, A. e Kronbauer, C. (2017). Intensidades das } \\
\text { intangibilidades e desempenhos econômico-financeiros em empresas dos países } \\
\text { do GLENIF. Revista de Gestão, Finanças e Contabilidade, 7(1). }\end{array}$ \\
\hline 29 & $\begin{array}{l}\text { Fuentes, N., Osorio, G. y Mungaray, A. (2016). Capacidades intangibles para la } \\
\text { competitividad microempresarial en México. Problemas del Desarrollo, 47(186). }\end{array}$ \\
\hline 30 & $\begin{array}{l}\text { Ahumada, E., Tovar, R., Perusquia, J. y Zárate, R. (2018). Gestión de } \\
\text { conocimiento en la competitividad del sector de TI. El caso de la región Tijuana - } \\
\text { San Diego. Sotavento M.B.A., 29, 30-40. }\end{array}$ \\
\hline 31 & $\begin{array}{l}\text { Ribeiro, V. e Vinícius, R. (2017). Análise dos efeitos do capital intelectual na } \\
\text { lucratividade das empresas brasileiras. Revista Universo Contábil, 13(4). }\end{array}$ \\
\hline 32 & $\begin{array}{l}\text { Talbot, L. y Colombo, A. (2019). Propiedad intelectual y } \\
\text { emprendimientos tecnológicos. Casos de emprendimientos de la } \\
\text { ciudad de Córdoba, Argentina. Revista Perspectiva Empresarial, 6(1), } 129 \text { - } 136 .\end{array}$ \\
\hline
\end{tabular}

Fuente: Elaboración propia

La intención en la revisión de los 32 artículos fue identificar las definiciones más representativas y utilizadas en el periodo ya especificado, y posteriormente continuar con la interpretación para articular los componentes de los activos intangibles con los elementos de la comunicación estratégica. Para lograr esta interpretación y construcción de sentido desde la perspectiva comunicacional, se seleccionaron las palabras o frases que se presentaron de manera consistente en las definiciones de los activos intangibles.

La contribución básica de esta técnica es la hermenéutica controlada basada en la inferencia. El esfuerzo de interpretación del análisis de contenido se mueve entre dos polos: el del rigor de la objetividad y el de la fecundidad de la subjetividad. De esta manera, ayuda a identificar lo latente, lo potencial y lo inédito encerrado en todo mensaje (Piñuel, 2002).

Este análisis se centra en la búsqueda del léxico que configura el contenido de las definiciones del concepto activos intangibles en producciones académicas. De tal manera que se logre identificar los tópicos intra e intertextuales, convergencias y divergencias 


\section{Revista Iberoamericana \\ de las Ciencias Sociales y \\ Humanísticas}

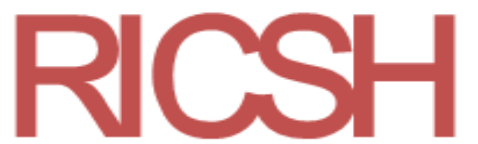

ISSN: $2395-7972$

desde la lógica de la comunicación estratégica, para hacer inferencias sobre las interrogantes que dieron origen a esta investigación: ¿para qué sirven los activos intangibles en la competitividad organizacional? y ¿cuáles son los elementos de la comunicación estratégica que intervienen en su proceso de producción?

Precisamente, definir el significado de las unidades de análisis constituye uno de los problemas fundamentales de esta técnica. Por lo que, para sortear esta dificultad, se utilizarán las categorías de cohesión léxica de Hoey (1991): repetición simple, repetición compleja, paráfrasis simple y la coreferencia.

Más adelante, se establecerán intratextualmente los agrupamientos léxicos, según la técnica de Phillips (1986): aquí se establecen las relaciones asociativas y cohesivas que perfilan la definición del tópico y que dan coherencia al significado. La técnica del agrupamiento léxico de Phillips (1986) sostiene que el texto revela la existencia de patrones sistemáticos que reflejan cierta organización. A través del análisis de conjuntos de palabras significativas, se configuran redes léxicas, las cuales se asocian con otras para articular gran parte de un área conceptual específica: los activos intangibles desde la mirada de la comunicación estratégica.

Finalmente, después de haber identificado las unidades léxicas más significativas, se establecen las coreferencias entre la definición de los activos intangibles y los componentes de la comunicación estratégica. Y se concluye con el registro de los términos centrales y periféricos articulados conceptual o funcionalmente a los componentes de la comunicación estratégica.

\section{Resultados}

Respecto a los elementos extratextuales, se puede mencionar que se obtuvieron 32 publicaciones académicas en Ebsco-Business Research, una base de datos de producción académica que presenta más de 282000 artículos de académicos y tiene una relación activa con más de 60000 editores de todo el mundo en los campos de ciencias de la vida, ciencias sociales, ciencias físicas y ciencias de la salud. Cabe señalar que este trabajo se enfocó en la búsqueda de publicaciones en el campo de los negocios. Ebsco-Business Research cubre tres tipos de fuentes: series de libros, revistas y revistas especializadas en el campo de los negocios, gestión y economía. Todas las revistas incluidas en esta base de datos mantienen altos estándares de calidad. En definitiva, de acuerdo con el número de publicaciones, se puede afirmar que la temática no es popular entre los autores que tienen un interés por la 


\section{Revista Iberoamericana \\ de las Ciencias Sociales y \\ Humanísticas}

ISSN: $2395-7972$

competitividad organizacional. Las 32 publicaciones representan $0.01 \%$ del total de la producción académica de la base de datos durante el periodo de análisis.

El periodo del estudio fue del 2016 al 2021, y durante estos cinco años se presentó una producción de seis artículos al año con la temática de activos intangibles. Por otra parte, se identificaron definiciones de un total de 22 autores (ver tablas 3 y 4 ).

Tabla 3. Conceptualización de intangibles (parte 1)

\begin{tabular}{|c|c|c|c|}
\hline Núm. & Autor & Año & Definición \\
\hline 1 & Kohler & 1983 & $\begin{array}{l}\text { Activo de capital que no tiene existencia física, cuyo valor } \\
\text { está limitado por los derechos y beneficios que la posesión } \\
\text { otorga a su dueño. }\end{array}$ \\
\hline 2 & Itami & 1987 & $\begin{array}{l}\text { Es la capacidad y habilidad de una empresa de incorporar } \\
\text { conocimiento en sus actividades productivas cotidianas. }\end{array}$ \\
\hline 3 & $\begin{array}{l}\text { Belkaoui } \\
\text { (citado en } \\
\text { Cañibano, } \\
\text { García y } \\
\text { Sánchez, } \\
\text { 1999) }\end{array}$ & 1992 & $\begin{array}{l}\text { Activos que carecen de sustancia física y que resultan de } \\
\text { derechos legales y contractuales; generan beneficios en el } \\
\text { futuro de forma probable. Incluyen la propiedad } \\
\text { intelectual, patentes y marcas. }\end{array}$ \\
\hline 4 & $\begin{array}{l}\text { Andersen } \\
\text { (citado en } \\
\text { Martínez, } \\
\text { 1996) }\end{array}$ & 1992 & $\begin{array}{l}\text { Aquellos recursos controlados por la empresa que no } \\
\text { tienen sustancia física que son capaces de generar en el } \\
\text { futuro beneficios económicos netos y están protegidos } \\
\text { legalmente o por medio de algún derecho de facto. }\end{array}$ \\
\hline 5 & Hall & 1992 & $\begin{array}{l}\text { Contratos, bases de datos, diseños y conocimientos de las } \\
\text { empresas relativos a la experiencia de los empleados, } \\
\text { cultura de la organización, etc. }\end{array}$ \\
\hline 6 & $\begin{array}{l}\text { Meigs, } \\
\text { Meigs, } \\
\text { Sinisterra y } \\
\text { Bedoya }\end{array}$ & 1993 & $\begin{array}{l}\text { Son aquellos que se usan en la operación de la empresa, } \\
\text { pero que no tiene sustancia física. Y no son corrientes. }\end{array}$ \\
\hline 7 & $\begin{array}{l}\text { Edvinsson } \\
\text { y Malone }\end{array}$ & 1999 & $\begin{array}{l}\text { La posesión de conocimientos, experiencia, tecnología, } \\
\text { relaciones con clientes y saberes profesionales que }\end{array}$ \\
\hline
\end{tabular}


Revista Iberoamericana

de las Ciencias Sociales y

Humanísticas

ISSN: $2395-7972$

\begin{tabular}{|l|l|l|l|}
\hline & & & $\begin{array}{l}\text { proveen a la empresa de ventaja competitiva en el } \\
\text { mercado. }\end{array}$ \\
\hline 8 & Stewart & 1998 & $\begin{array}{l}\text { Son aquellos que poseen valor sin tener dimensiones } \\
\text { físicas_y están localizados en las personas (empleados, } \\
\text { clientes, proveedores), o bien se obtienen a partir de } \\
\text { procesos, sistemas y la cultura organizativa. }\end{array}$ \\
\hline 9 & $\begin{array}{l}\text { Fernández } \\
\text { et. al. }\end{array}$ & 1998 & $\begin{array}{l}\text { Consisten en conocimiento e información y pueden } \\
\text { constituir una diferencia competitiva. Conocimiento, } \\
\text { cultura y tecnología y reputación. }\end{array}$ \\
\hline 10 & $\begin{array}{l}\text { Hendriksen } \\
\text { y Van }\end{array}$ & 1999 & $\begin{array}{l}\text { Activos que carecen de sustancia. Como tales, estos } \\
\text { activos deben reconocerse siempre que cumplan con la } \\
\text { definición de activos (ser mensurables, pertinentes y } \\
\text { exactos). }\end{array}$ \\
\hline 11 & Lev & 2001 & $\begin{array}{l}\text { Son un derecho a los beneficios futuros que no tienen } \\
\text { cuerpo físico o financiero, que se crea por la innovación, } \\
\text { prácticas de organización y recursos humanos. Los activos } \\
\text { intangibles interactúan con los activos tangibles en la } \\
\text { creación de valor corporativo y el crecimiento económico. }\end{array}$ \\
\hline
\end{tabular}

Fuente: Elaboración propia 


\section{Revista Iberoamericana \\ de las Ciencias Sociales y \\ Humanísticas}

ISSN: 2395 - 7972

Tabla 4. Conceptualización de activos intangibles (parte 2)

\begin{tabular}{|c|c|c|c|}
\hline Núm. & Autor & Año & Definición \\
\hline 12 & Upton & 2001 & $\begin{array}{l}\text { Recursos no físicos generadores de probables beneficios } \\
\text { económicos futuros para la entidad que fueron } \\
\text { adquiridos a través del intercambio o desarrollados } \\
\text { internamente sobre la base de costos identificables, que } \\
\text { tienen una vida limitada, que poseen valor de mercado } \\
\text { propio y que pertenecen y son controlados por la } \\
\text { entidad. }\end{array}$ \\
\hline 13 & Kayo & 2002 & $\begin{array}{l}\text { Conjunto estructurado de conocimientos, prácticas y } \\
\text { actitudes de la empresa que, interactuando con sus } \\
\text { activos tangibles, contribuye a la formación de valor de } \\
\text { esta. }\end{array}$ \\
\hline 14 & Navas y Ortiz & 2002 & $\begin{array}{l}\text { Son aquellos activos que no tienen soporte físico, ya que } \\
\text { están basados en la información y en el conocimiento. }\end{array}$ \\
\hline 15 & $\begin{array}{l}\text { Kaplan y } \\
\text { Norton }\end{array}$ & 2004 & $\begin{array}{l}\text { Las capacidades, habilidades, conocimientos o formas de } \\
\text { trabajar; pueden impulsar que los clientes vean } \\
\text { satisfechas sus necesidades y expectativas y los } \\
\text { resultados financieros acaben llegando. }\end{array}$ \\
\hline 16 & $\begin{array}{l}\text { Universidad } \\
\text { EAFIT }\end{array}$ & 2006 & $\begin{array}{l}\text { Se entiende como activo intangible todo aquel } \\
\text { identificable, de carácter no monetario, y sin apariencia } \\
\text { física, que se posee para ser utilizado en la producción o } \\
\text { suministro de bienes y servicios, para ser arrendado a } \\
\text { terceros o para funciones relacionadas con la } \\
\text { administración de la entidad. }\end{array}$ \\
\hline 17 & $\begin{array}{l}\text { Miles y } \\
\text { Quintillán }\end{array}$ & 2005 & $\begin{array}{l}\text { Conjunto de capacidades (conocimiento y } \\
\text { competencias), motivaciones, elementos culturales y } \\
\text { otros del estilo que posee la empresa en un momento } \\
\text { determinado. Son recursos no monetarios, sin sustancia } \\
\text { física, que en combinación son capaces de producir } \\
\text { beneficios para una compañía. }\end{array}$ \\
\hline 18 & Sinisterra, & 2011 & Estos activos implican un derecho o privilegio y de cuyo \\
\hline
\end{tabular}




\section{Revista Iberoamericana \\ de las Ciencias Sociales y \\ Humanísticas}

ISSN: $2395-7972$

\begin{tabular}{|c|c|c|c|}
\hline & $\begin{array}{l}\text { Polanco y } \\
\text { Henao }\end{array}$ & & $\begin{array}{l}\text { ejercicio o explotación pueden obtenerse beneficios } \\
\text { económicos en varios periodos determinantes. }\end{array}$ \\
\hline 19 & $\begin{array}{l}\text { Blázquez y } \\
\text { Amato }\end{array}$ & 2011 & $\begin{array}{l}\text { Son los recursos humanos: conocimientos, habilidades, } \\
\text { capacidades, experiencias; son los recursos tecnológicos: } \\
\text { grado de tecnología alcanzado; administrativos: } \\
\text { propiedad intelectual, marcas, patentes; y culturales: } \\
\text { valores corporativos, identidad e imagen institucional }\end{array}$ \\
\hline 20 & $\begin{array}{l}\text { Cuéllar, } \\
\text { Vargas y } \\
\text { Castro }\end{array}$ & 2012 & $\begin{array}{l}\text { Aquellos que no tienen forma física, pero sobre los } \\
\text { cuales la entidad posee derechos y espera recibir } \\
\text { beneficios futuros. }\end{array}$ \\
\hline 21 & $\begin{array}{l}\text { Organización } \\
\text { para la } \\
\text { Cooperación } \\
\text { y el } \\
\text { Desarrollo } \\
\text { Económicos } \\
\text { (OCDE) }\end{array}$ & 2013 & $\begin{array}{l}\text { De naturaleza incorpórea, genera beneficios futuros a la } \\
\text { organización, tal y como derechos de utilización de } \\
\text { activos industriales: patentes, marcas comerciales, } \\
\text { nombres comerciales, dibujos, modelos; propiedad } \\
\text { intelectual, literaria y artística. }\end{array}$ \\
\hline 22 & $\begin{array}{l}\text { Consejo } \\
\text { Mexicano de } \\
\text { Normas de } \\
\text { Información } \\
\text { Financiera } \\
\text { [Cinif] }\end{array}$ & 2014 & $\begin{array}{l}\text { Los intangibles son activos no monetarios y representan } \\
\text { generalmente derechos abstractos, como puedes ser una } \\
\text { patente, una licencia de uso, una franquicia, una imagen } \\
\text { de marca que crea una relación especial con los clientes, } \\
\text { derechos de autor. }\end{array}$ \\
\hline
\end{tabular}

Elaboración propia

Posteriormente, se establecieron intratextualmente los agrupamientos léxicos, según la técnica de Phillips (1986). El primer agrupamiento que se identifica en el análisis de las definiciones de los activos intangibles responde a la perspectiva económica y financiera, debido a que los elementos fundamentales para la producción de bienes y servicios estaban cimentados en tres pilares tradicionales: los recursos materiales, financieros y tecnológicos. En la figura 1 se observa que aún persiste la tendencia de abordajes financieros y contables (19\%). 


\section{Revista Iberoamericana \\ de las Ciencias Sociales y \\ Humanísticas}

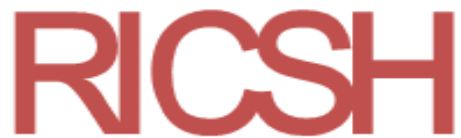

ISSN: 2395 - 7972

Figura 1. Enfoques conceptuales

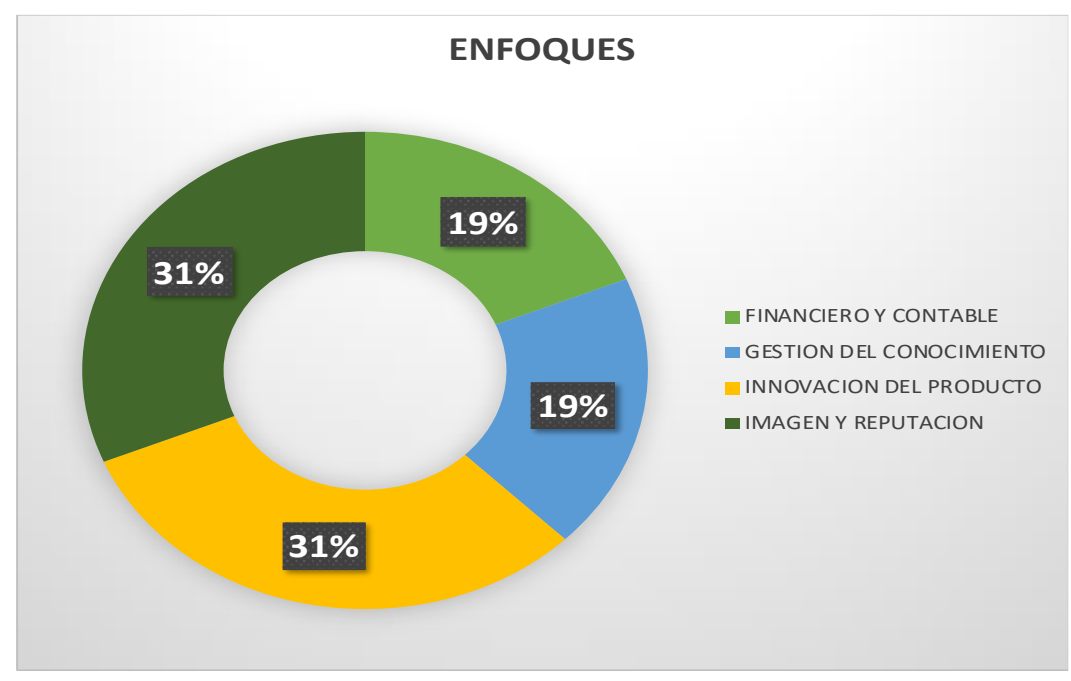

Fuente: Elaboración propia

Sin embargo, las dinámicas organizacionales contemporáneas giran en torno a la incorporación de las tecnologías de comunicación e información (TIC), los procesos de globalidad económica, así como los cambios en los patrones de consumo de las personas que han transformado la oferta de los bienes y servicios, lo que ha dado lugar a una sociedad donde se privilegian el conocimiento y la información como elementos consustanciales de la competitividad. En la figura 2 se observa el despunte del abordaje de la innovación de producto, con $31 \%$. Además, el enfoque orientado a la gestión del conocimiento (19\%) ocupa un lugar por encima del tradicional enfoque contable. Es importante destacar que estos enfoques se orientan a la concepción de los activos intangibles como resultado de procesos relacionales y simbólicos en los que se requiere del intercambio de significados y sentidos de manera transversal en la dinámica organizacional, principios fundamentales de la comunicación estratégica.

Por otra parte, existe consenso sobre la cualidad no corpórea de los activos intangibles; sin embargo, aun con esta coincidencia, se presenta una dispersión en cuanto al término que acompaña a la cualidad, pues se combina con vocablos como apariencia, soporte, sustancia, existencia o naturaleza. Esta dispersión no es menor, ya que cambia el significado de la intangibilidad en relación con cada una de las diferentes dimensiones. Estas relaciones se observan en la figura 2, donde se enlista la diversidad de combinaciones que los autores utilizan para describir a los activos intangibles. 


\section{Revista Iberoamericana \\ de las Ciencias Sociales y \\ Humanísticas}

ISSN: 2395 - 7972

Figura 2. Características y cualidades

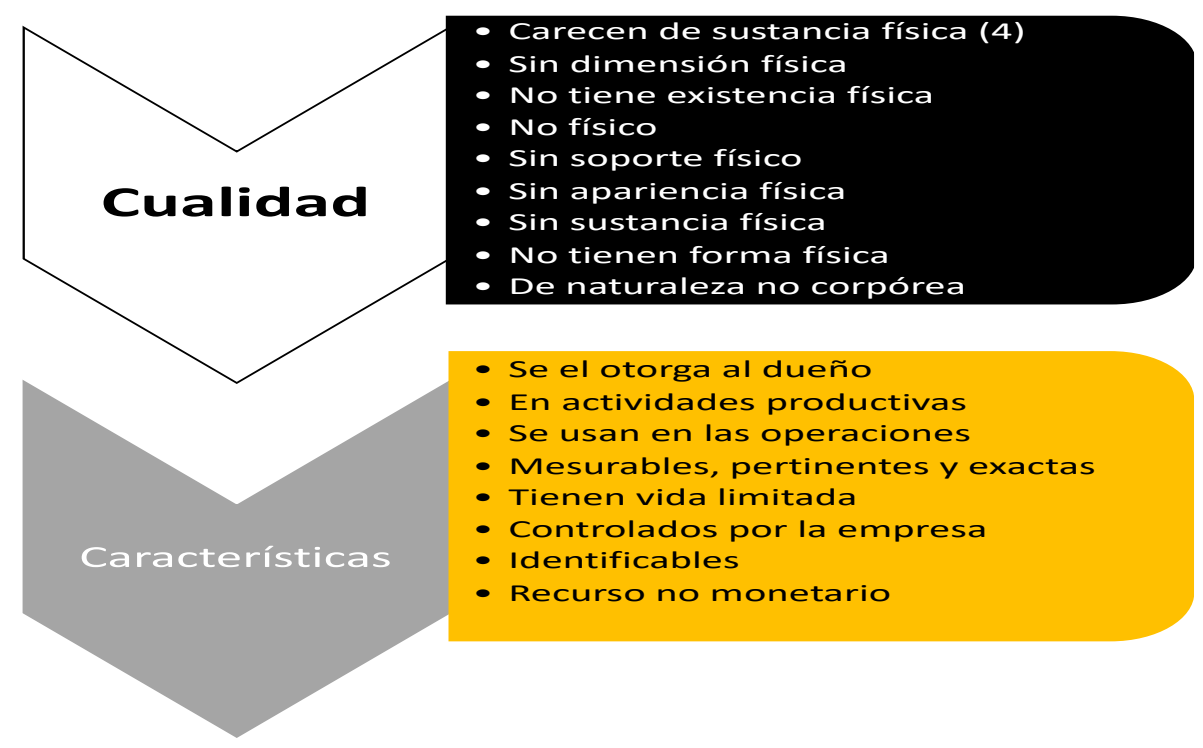

Fuente: Elaboración propia

Aunado a lo anterior, siguiendo los resultados en torno a la descripción de sus características que se presentan en la figura 2, no se identificó ninguna frecuencia o redundancia significativa. En el análisis de este rubro es interesante destacar que la definición sobre la posesión de los activos intangibles no queda claramente definida. Se coincide con sus características de vida limitada y el interés por ser controlados por la organización para orientarlos hacia los objetivos organizacionales, hay consenso en torno al impacto en los resultados financieros, sin embargo, no se presentan claramente los indicadores a considerar para cuantificar este beneficio.

En este mismo sentido, algunos autores coinciden en el valor que representan para la empresa en general, al igual que en la injerencia que tienen sobre los resultados económicos de la organización, por lo cual su gestión es de suma importancia, pues genera múltiples beneficios en el ámbito financiero. También existe convenio entre los autores sobre el impacto que tienen en la competitividad de la organización, aunque no se manifiestan de manera directa y a corto plazo, por lo que es necesario mantener procesos de gestión de estos activos de manera consistente para lograr identificar los resultados significativos en los objetivos organizacionales. En la figura 3 se muestran los términos con los cuales los autores identifican su origen e impacto. Aquí también existe un amplio consenso. 


\section{Revista Iberoamericana \\ de las Ciencias Sociales y Humanísticas}

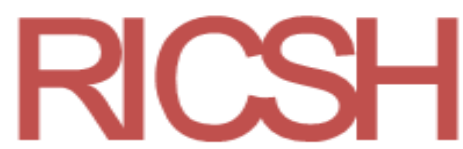

ISSN: 2395 - 7972

Figura 3. Origen e impacto

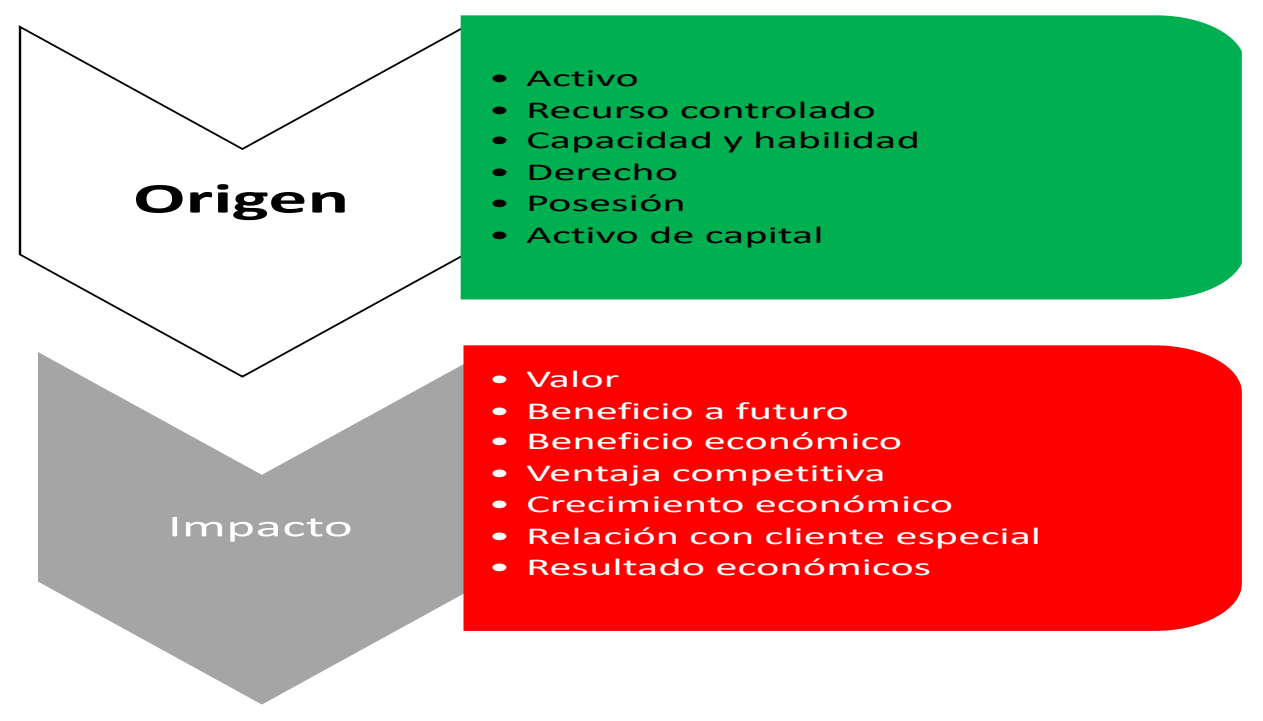

Fuente: Elaboración propia

Sin embargo, la traducción o aplicación de estos beneficios tiene sus divergencias, ya que depende de la mirada desde la cual se construyen los indicadores de medición. Desde la contabilidad financiera, los beneficios proceden de títulos de propiedad, derechos contractuales, patentes, derechos de propiedad intelectual, entre otros. Desde esta perspectiva, existen métricas financieras internacionales para el registro y evaluación de dichos activos postuladas por organismos internacionales como el Cinif (2014).

Desde la perspectiva comunicacional, en los últimos años la concepción de los activos intangibles se ha orientado hacia la marca, la reputación corporativa, la ética empresarial, la responsabilidad social, el buen gobierno, entre otros. Hoy estos elementos son vitales y estratégicos para asegurar el éxito de las organizaciones en el entorno global (López, 2011). En la figura 4 se observan los términos usados en torno a la categoría de componentes de los activos intangibles, que van desde información, conocimiento, destrezas hasta cultura, relación con los clientes o percepción del mercado; y en menor medida, plantea los beneficios sobre ejercicios contractuales y legales. 


\section{Revista Iberoamericana \\ de las Ciencias Sociales y \\ Humanísticas}

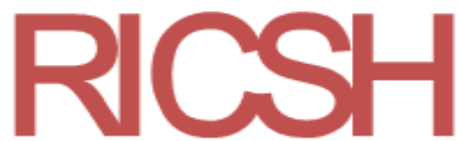

ISSN: 2395 - 7972

Figura 4. Componentes de los activos intangibles

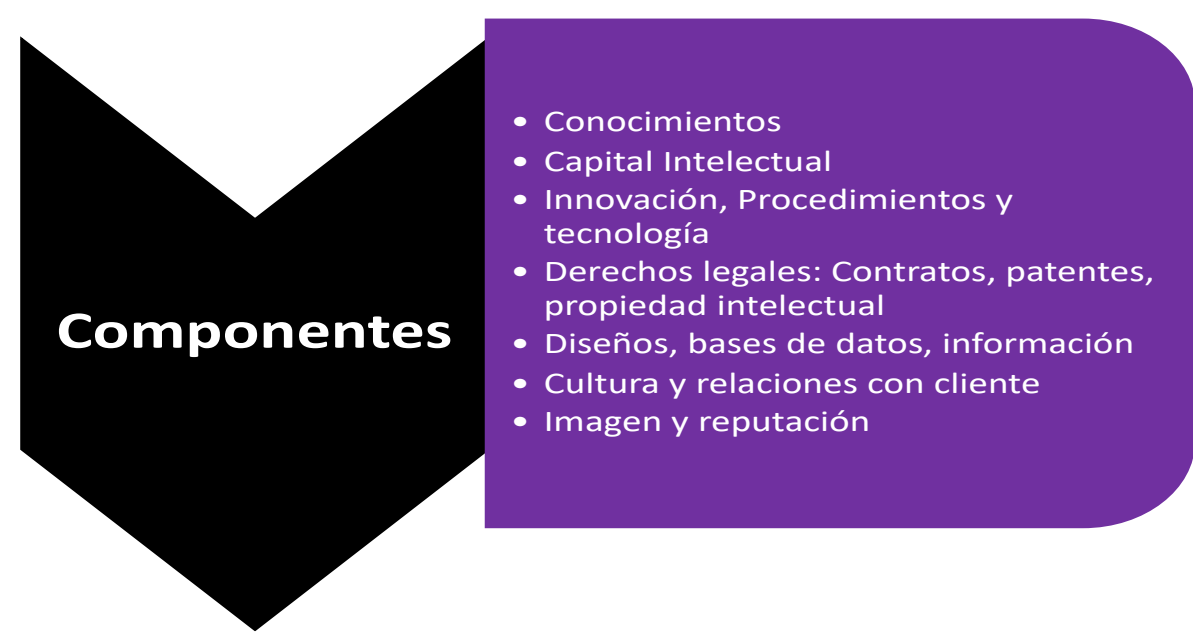

Fuente: Elaboración propia

La tendencia dominante se observa hacia el conocimiento, la innovación en las actividades de producción, la distribución y el consumo; además, se identifica una tendencia para vincular a los activos intangibles con otros factores que detonan la productividad, como procesos de producción, interacciones de las empresas con sus usuarios y la diversificación en la oferta de productos, entre otros (Vilaseca, Torrent y Díaz, 2002).

\section{Discusión}

Después de haber identificado las unidades léxicas más significativas, se determinó la coreferencia con la perspectiva de la comunicación estratégica. La comunicación estratégica es concebida como un proceso generador de energía simbólica, movimiento relacional y acción social, por lo que su función estratégica es la generación de activos intangibles dentro del espectro organizacional. Partiendo de esta perspectiva comunicacional, es importante considerar que los activos intangibles están ligados a una dimensión macrosistémica de la organización, y por ello otorgan valor a los resultados estratégicos, y no a los resultados operativos.

El paradigma de la comunicación estratégica es resultante, por una parte, del esfuerzo operativo de transmitir información; por otra, del esfuerzo interactivo con sus grupos de interés, que provoca un nivel de satisfacción y efectividad organizacional; pero, fundamentalmente, del esfuerzo de la mejora del sistema que desencadena en un proceso de innovación que construye una red de valor compartido. Y por ello, la finalidad estratégica 


\section{Revista Iberoamericana \\ de las Ciencias Sociales y \\ Humanísticas}

ISSN: $2395-7972$

de la comunicación se orienta a la producción de activos intangibles que potencialicen los resultados funcionales de la organización y que trascienda en el entorno social.

Para identificar los elementos de coreferencia de los activos intangibles con la perspectiva de la comunicación estratégica, se partió de la tipología que propone López (2011). Como resultado, en la tabla 5 se señalan los tópicos significativos y que presentan una coreferencia con los componentes identificados en las definiciones de los activos intangibles presentadas en el apartado anterior.

Tabla 5. Coreferencia de los activos intangibles con la Comunicación Estratégica

\begin{tabular}{|l|l|}
\hline Capital humano & $\begin{array}{l}\text { Recoge las capacidades individuales, los conocimientos, la } \\
\text { destreza y la experiencia de los empleados. }\end{array}$ \\
\hline Capital estructural & $\begin{array}{l}\text { Infraestructura que incorpora, forma y sostiene al capital humano. } \\
\text { Se define como el conjunto de conocimientos que permanece en } \\
\text { la empresa al final de la jornada laboral. Comprende las rutinas } \\
\text { organizativas, los procedimientos, los sistemas, las culturas, las } \\
\text { bases de datos, entre otras. Está compuesto por tres tipos de } \\
\text { capital: }\end{array}$ \\
$\begin{array}{l}\text { 1) Capital organizacional: inversión de la empresa en sistemas, } \\
\text { herramientas y filosofía operativa }\end{array}$ \\
$\begin{array}{l}\text { 2) Capital de innovación: capacidad de renovación y resultados de } \\
\text { la innovación en forma de derechos comerciales protegidos, } \\
\text { propiedad intelectual y otros activos intangibles usados para crear } \\
\text { nuevos productos y servicios. }\end{array}$ \\
3) Capital de clientela: relaciones de la empresa con clientes \\
medidas a través de índices de satisfacción o longevidad, entre \\
otros.
\end{tabular}




\section{Revista Iberoamericana}

de las Ciencias Sociales y

Humanísticas

ISSN : $2395-7972$

\begin{tabular}{|c|l|}
\hline Responsabilidad & Está compuesta por compromisos en la gestión de una empresa. \\
social corporativa & $\begin{array}{l}\text { Son realidades y residen en la empresa. Esta responsabilidad es } \\
\text { unos de los varios factores que influyen en la reputación } \\
\text { corporativa }\end{array}$ \\
\hline
\end{tabular}

Fuente: Elaboración propia con base en López (2011)

La coreferencia de las características de los activos intangibles con la perspectiva de la comunicación estratégica se orientan en tres sentidos:

1) La comunicación como un proceso en el que se articulan los recursos informativos, se desarrollan procesos de aprendizaje organizacional y se fortalece la capacidad detección de necesidades del entorno. Por lo tanto, es un proceso de transferencia en la generación de capital intelectual.

2) La comunicación como un proceso en el que se fortalecen las relaciones entre la organización y sus grupos de interés; ayuda a responder de manera más pertinente y oportuna a las demandas del entorno. En suma, se trata de un proceso de interacción en la generación de capital relacional.

3) La comunicación como un proceso de gestión de los recursos sociales, relacionales y perceptuales que permite crear un ecosistema simbólico que servirá de plataforma para dinamizar los procesos funcionales y operativos de la organización. Es un proceso perceptual que genera capital simbólico.

Partir de la función estratégica del sistema de comunicación en la organización es dimensionar el papel articulador de los procesos sociales con los propósitos de la estrategia corporativa. En este sentido, el sistema comunicacional debe buscar acoplar las dimensiones estructurales, funcionales, relacionales y simbólicas de la organización de tal forma que genere valor en todos los componentes de la red. Esto significa interpretar la producción simbólica de la organización, escuchando los discursos colectivos, articulando los patrones de comportamiento, acoplando las representaciones sociales, respondiendo a las demandas de los múltiples escenarios que interactúan en el contexto, para detonar procesos de mediación en la organización y de adaptación a nivel social.

La interpretación de la producción simbólica del sistema social va más allá del contenido del mensaje; implica el reconocimiento del significado y el sentido que los agentes organizacionales le confieren a la situación, y que en muchas ocasiones se expresan en los intangibles organizacionales. Kaplan y Norton (2004) los categorizan en tres niveles, como se puede apreciar en la figura 5. 


\section{Revista Iberoamericana \\ de las Ciencias Sociales y \\ Humanísticas}

ISSN: $2395-7972$

Figura 5. Los activos intangibles

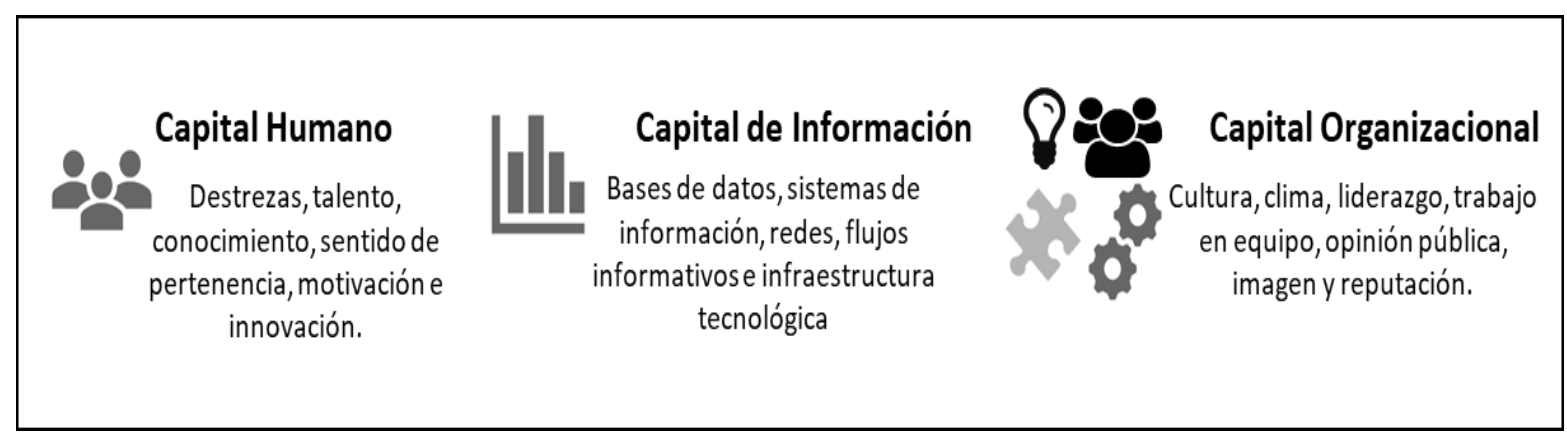

Fuente: Kaplan y Norton (2004)

Por su trascendencia, los activos intangibles deben ser gestionados por la organización de tal forma que se conviertan en una plataforma simbólica que potencialice los resultados corporativos. Sin embargo, por su composición multifactorial y dimensional, no pueden ser gestionados desde una perspectiva lineal, determinista y de causa-efecto; por su construcción compleja, los intangibles no generan valor por sí mismos, para que se manifieste su valor deben ser acompañados de otros activos organizacionales, y de esta forma convertirse en catalizadores de las funciones prioritarias de la organización (Kaplan y Norton, 2004).

Ahora bien, para poder articular a la comunicación estratégica con los activos intangibles, se debe apuntar a la transversalidad de la producción simbólica de la organización. Reygadas (2002), al referir las aportaciones de Lupton, sugiere que los integrantes de una organización tienen una carga simbólica de origen que moldea su comportamiento individual, y esto a su vez le da forma al comportamiento y a la cultura organizacional. Por ello, como se muestra en la figura 6, es importante crear redes y ambientes ricos en información que apoyen los objetivos organizacionales, como lo plantea Nosnik (2013), y que logren armonizar la producción simbólica individual con la operativa, funcional y corporativa. 


\section{Revista Iberoamericana \\ de las Ciencias Sociales y \\ Humanísticas}

ISSN: $2395-7972$

Figura 6. La comunicación estratégica en la generación de valor organizacional

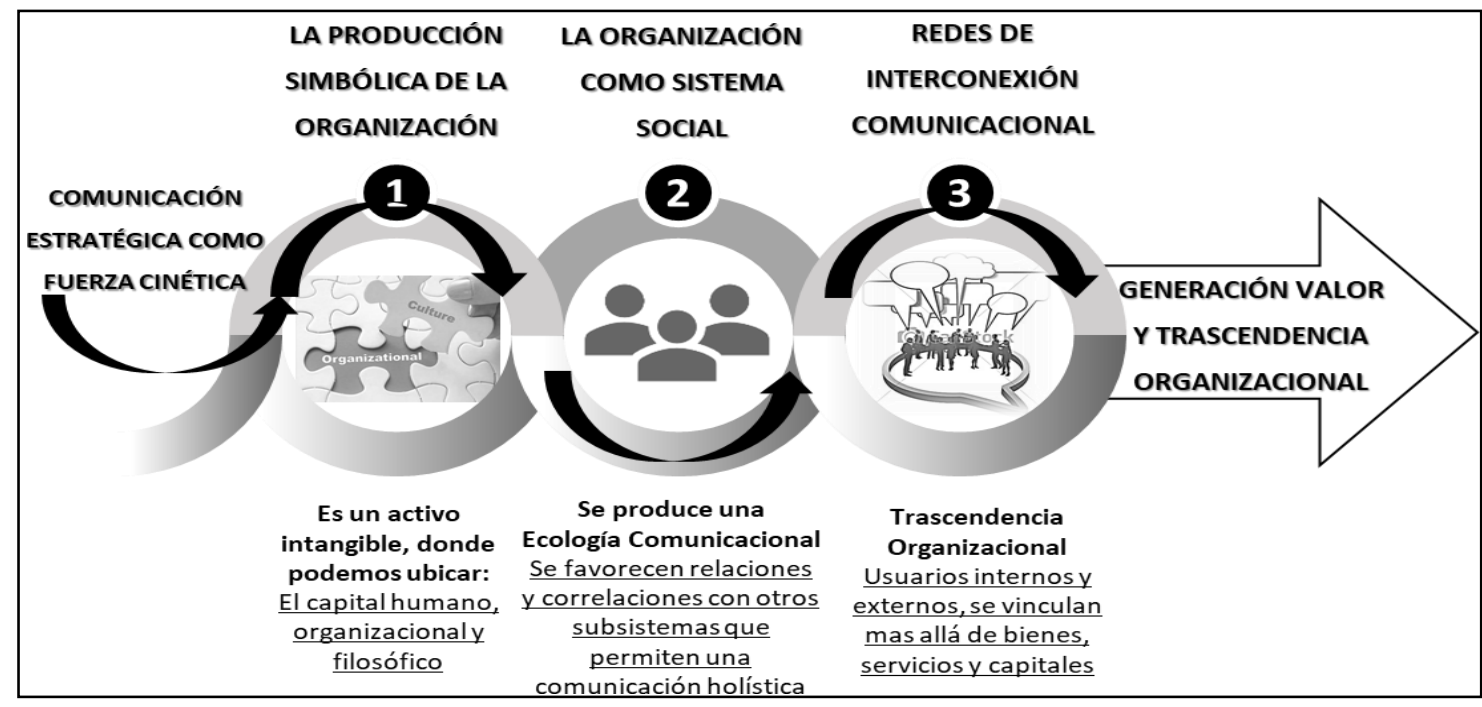

Fuente: Elaboración propia

\section{Conclusiones}

Para dar respuesta a las interrogantes que motivaron este análisis se puede mencionar que los autores estudiados coinciden en la importancia y trascendencia que representan los activos intangibles para la competitividad organizacional. Por otra parte, se puede identificar que la tendencia dominante por décadas para fundamentar el valor de las organizaciones sobre su capacidad para producir se encuentra en descenso. Los planteamientos en donde la competitividad organizacional estaba asociada únicamente a la cantidad de recursos económicos, a la estandarización de su producción, a la división racional del trabajo, ahora se combinan con otros enfoques y perspectivas.

Con las modificaciones en los escenarios organizacionales se ha fortalecido la concepción del valor de las corporaciones más orientadas a su capacidad para saber hacer, sus relaciones sociales y la percepción de sus grupos de interés. Lo que posiciona a los activos intangibles como componentes estratégicos que otorgan diferenciación competitiva a las organizaciones.

Por otra parte, la revisión sobre los elementos de la comunicación estratégica que se vinculan con la generación de los activos intangibles proporciona respuestas aún más complejas y trascendentes. En un sistema organizacional, la comunicación es una función que dinamiza, orienta y maximiza las interacciones; además, potencializa la capacidad relacional, la construcción en colectivo y el logro de metas de largo plazo. Por lo que su 


\section{Revista Iberoamericana \\ de las Ciencias Sociales y \\ Humanísticas}

ISSN: $2395-7972$

función estratégica es la generación de activos intangibles que le otorguen un soporte simbólico a la competitividad organizacional.

Sin embargo, es importante señalar que esta aportación simbólica que se produce en la dinámica organizacional se inserta en los procesos sociales que trascienden el ámbito corporativo, por lo que la producción de activos intangibles a raíz de procesos comunicacionales trae como resultado valor competitivo y riqueza social. Desde la perspectiva de la comunicación estratégica, intervenir en las organizaciones es una opción que tienen los seres humanos para modificar las estructuras del entorno social. Por lo que, en la medida en que se generen espacios de entendimiento y coordinación en las organizaciones para la construcción de activos intangibles, se puede contribuir a la generación de dinámicas sociales más orientadas a la acción colectiva sustentable basada en el diálogo.

En este sentido, la comunicación estratégica alcanza su máximo nivel en una organización cuando logra generar espacios vinculantes que propician innovación, diferenciación y competitividad. Para lograrlo, se requiere de un conjunto de esfuerzos colectivos sintetizados en procesos de transmisión, intercambio y apropiación para convertirse en un sistema productivo de convivencia social. Además, se requiere considerar que cualquier entidad organizativa posee recursos, competencias particulares y capacidades específicas que deberán capitalizarse para contribuir de manera significativa a la competitividad organizacional. Así pues, es necesario considerar que los activos intangibles no generan valor por sí mismos, sino que para que adquieran valía deben venir acompañados de otros activos organizacionales.

En este sentido, desde la perspectiva comunicacional se propone considerar las siguientes relaciones. En primer lugar, lo que se hace y cómo se hace, que se traduce en los productos y servicios con calidad y elementos competitivos. El activo intangible se encuentra en el sello distintivo que le otorga el comportamiento o cultura corporativa a ese producto o servicio.

En segundo lugar, lo que se dice y cómo se dice, que implica considerar todos los momentos expresivos de la organización en una estrategia global de comunicación que apoye la estrategia corporativa. En este caso, el activo intangible se genera cuando se logra vincular la producción simbólica de los grupos de interés con la organización y se traduce en una marca posicionada o imagen corporativa fuerte. 


\section{Revista Iberoamericana \\ de las Ciencias Sociales y \\ Humanísticas}

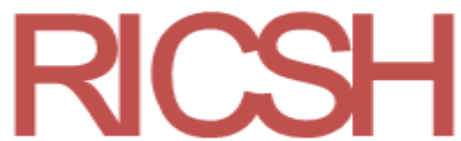

ISSN: 2395 - 7972

Finalmente, para qué lo haces y para qué lo dices, expresado en los objetivos, la filosofía corporativa y la responsabilidad social. El activo intangible se traduce a largo plazo en el compromiso y relación sustentable de la organización con todos sus grupos de interés que le aportan valor estratégico. Estos activos intangibles se conocen como reputación, posicionamiento e innovación.

\section{Futuras líneas de investigación}

Para alcanzar un nivel estratégico de la competitividad organizacional se requiere un esfuerzo sistematizado en la generación de activos intangibles. Asimismo, es necesario desarrollar líneas de investigación en las que se profundice sobre los procesos psicológicos, cognitivos y sociales que intervienen en la construcción de las ecologías entre la organización, sus grupos de interés y el entorno. Por otra parte, resulta interesante explorar sobre las herramientas de gestión que apoyen la articulación de las dimensiones entre lo que se dice y se hace, es decir, herramientas informativas y de actuación. Finalmente, es importante impulsar la producción de conocimiento que logre fortalecer la relación e influencia que tienen los activos tangibles e intangibles de tal manera que se potencialice la competitividad. 


\section{Revista Iberoamericana \\ de las Ciencias Sociales y \\ Humanísticas}

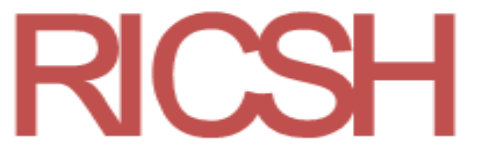

ISSN: 2395 - 7972

\section{Referencias}

Blázquez, M. y Amato, C. (2011). CORPRIC: Hacia un nuevo diagnóstico para la gestión organizacional. Ponencia presentada en el XXVII Congreso de Docentes de Universidades en Administración General. Santa Rosa, 2011.

Cañibano, L. García, M. y Sánchez, M. P. (1999). La relevancia de los intangibles por la valoración y la gestión de empresa: revisión de la literatura. Revista Española de Financiación y Contabilidad, (100).

Cuéllar, L., Vargas, H. y Castro, C. (2012). Contabilidad, un enfoque práctico. Colombia: Alfaomega.

Consejo Mexicano de Normas de Información Financiera [Cinif]. (2014). Norma de Información Financiera A-5. Consejo Mexicano de Normas de Información Financiera.

Edvinsson, L. y Kivikas, M. (2004). La nueva perspectiva para la creación de valor. Revista de Contabilidad y Dirección, 1, 47-57.

Edvinsson, L. y Malone, M. S. (1999). El capital intelectual. Barcelona, España: Gestión 2000.

Hall, R. (1992). The Strategic Analysis of Intangible Resources. Strategic Management Journal, 13(2), 135-144.

Hendriksen, E. y Van Breda, M. (1999). Teoria da contabilidade. São Paulo. Editorial Atlas S.A.

Hoey, M. (1991). Patterns of Lexis in Text. Oxford, England: Oxford University Press.

Itami, H. (1987). Mobilizing Invisible Assets. Cambridge, United States: Harvard University Press.

Kaplan, R. S. y Norton D. P. (2004) Medir la disposición estratégica de los activos intangibles. Harvard Business Review, 82(2), 42-53.

Kaplan, R. and Norton, D. P. (2003). Strategy Maps: Converting Intangible Assets into Tangible Outcomes ( $1^{\text {st }}$ ed.). Boston, United States: Harvard Business School.

Kayo, E. K. (2002). A estrutura de capital e o risco das empresas tangível e intangível intensivas: uma contribuição ao estudo da valoração das empresas. (Tese de doutorado). Universidade de São Paulo, São Paulo.

Kohler, E. L. (1983). Dictionary for Accountants (6 ${ }^{\text {th }}$ ed.). Englewood Cliffs, United States: Prentice-Hall. 


\section{Revista Iberoamericana \\ de las Ciencias Sociales y \\ Humanísticas}

ISSN: $2395-7972$

Lev, B. (2001). Intangibles: Management, Measurement and Reporting. Washington, United States: Brookings Institution Press.

Lev, B. (2003). Intangibles: medición, gestión e información. Barcelona, España: Ediciones Deusto.

López, A. (2011). La gestión de activos y recursos intangibles: una oportunidad para las empresas, los gerentes de riesgos y el mercado asegurador. Gerencia de Riesgos y Seguros, 28(110), 46-60.

Martínez, L. (1996). Activos intangibles e información contable. Ponencia presentada en el VIII Encuentro de Profesores Universitarios de Contabilidad. Barcelona, del 5 al 8 de junio de 1996.

Meigs, R., Meigs, W., Sinisterra, G. y Bedoya, O. (1993). Contabilidad: la base para decisiones comerciales (3. ${ }^{\mathrm{a}}$ ed.). Bogotá, Colombia: McGraw-Hill.

Miles, J. y Quintillán, I. (2005). Auto-evaluación del capital intelectual en la pequeña y mediana empresa. Revista Electrónica FCN.

Navas, E. y Ortiz, M. (2002). El capital intelectual en la empresa. Análisis de criterios y clasificación multidimensional. Economía Industrial, 4(346), 163-171.

Nosnik, A. (2013). Teoría de la comunicación productiva (1. ${ }^{\mathrm{a}}$ ed.). Argentina: Homo Sapiens.

Organización para la Cooperación y el Desarrollo Económicos [OECD]. (2021). Estrategia de innovación de la OCDE: llevarle ventaja al mañana. Organización para la Cooperación y el Desarrollo Económicos. Recuperado de https://www.oecd.org/sti/45302715.pdf.

Phillips, M. (1989). Lexical Structure of Text. Birmingham, England: University of Birmingham.

Piñuel, J. L. (2002). Epistemología, metodología y técnicas del análisis de contenido. Estudios de Sociolingüística, 3(1), 1-42.

Reygadas, L. (2002) Producción simbólica y producción material: metáforas y conceptos en torno a la cultura del trabajo. Nueva Antropología, 17(60). Recuperado de http://www.redalyc.org/articulo.oa?id=15906007.

Sinisterra, P., Polanco, L. E. y Henao, H. (2011). Contabilidad sistema de información para las organizaciones (6. ${ }^{\mathrm{a}} \mathrm{ed}$.). Bogotá, Colombia: McGraw-Hill.

Stewart, T. A. (1998). La nueva riqueza de las organizaciones: el capital intelectual. Barcelona, España: Granica. 


\section{Revista Iberoamericana \\ de las Ciencias Sociales y \\ Humanísticas}

ISSN: 2395 - 7972

Universidad EAFIT. (s. f.). NIC 38 - Activos intangibles.

Upton, W. S. (2001). Business and Financial Reporting. Challenges from the New Economy. Norwalk, United States: Financial Accounting Standards Board.

Vilaseca, J., Torrent, J. y Díaz, Á. (2002). La economía del conocimiento: paradigma tecnológico y cambio estructural: un análisis empírico e internacional para la economía española. (Documento de trabajo). Universitat Oberta de Catalunya, Barcelona.

\begin{tabular}{|l|l|}
\hline Rol de Contribución & Autor (es) \\
\hline Conceptualización & Patricia Durán Bravo \\
\hline Metodología & Principal Patricia Durán Bravo, que apoya Víctor Meléndez \\
\hline Software & No aplica \\
\hline Validación & Igual Patricia Durán Bravo, Víctor Meléndez \\
\hline Análisis Formal & Principal Patricia Durán Bravo, que apoya Víctor Meléndez \\
\hline Investigación & Patricia Durán Bravo, \\
\hline Recursos & Igual Víctor Meléndez Rafael Cid Quiroz \\
\hline Curación de datos & Principal. Patricia Durán Bravo que apoya Víctor Meléndez \\
\hline $\begin{array}{l}\text { Escritura - Preparación del } \\
\text { borrador original }\end{array}$ & Principal. Patricia Durán Bravo que apoya Víctor Meléndez \\
\hline $\begin{array}{l}\text { Escritura - Revisión y } \\
\text { edición }\end{array}$ & Patricia Durán Bravo. \\
\hline Visualización & Rafael Cid Quiroz \\
\hline Supervisión & Igual Patricia Durán Bravo, Víctor Meléndez \\
\hline Administración de Proyectos & Igual Patricia Durán Bravo, Víctor Meléndez \\
\hline Adquisición de fondos & N.A. \\
\hline
\end{tabular}

